\title{
Diagnóstico y manejo oportuno del síndrome de HELLP
}

\author{
Pablo Parra- Ramírez, Mario Beckles- Maxwell
}

\section{Resumen}

El síndrome de HELLP (SH) que según sus siglas en inglés consiste en hemólisis, aumento enzimas hepáticas y trombocitopenia, es una de las complicaciones maternas y fetales más graves durante el embarazo. El 10\% de los embarazos complicados con preeclampsia severa o eclampsia son afectados por el SH. Este síndrome ha sido descrito desde la mitad del segundo trimestre hasta varios días pos-parto. Aproximadamente un tercio de los pacientes van a desarrollar sus primeras manifestaciones de SH hasta varios días posparto. De los dos tercios de mujeres que son diagnosticadas con $\mathrm{SH}$ en el anteparto, el $10 \%$ se presentan antes de las 27 semanas, el $20 \%$ luego de las 37 semanas y el $70 \%$ ocurren entre las 27 y 37 semanas de gestación. La mortalidad materna ha sido reportada en un $24 \%$ y la perinatal en un $30-40 \%$. El diagnóstico y manejo temprano del SH resulta un problema para los profesionales en obstetricia, esto debido principalmente a la falta de signos y síntomas específicos, lo cual retarda el adecuado manejo de ésta patología si no se diagnostica en sus inicios o adecuadamente. El manejo de embarazos complicados con el $\mathrm{SH}$ requiere de un reconocimiento temprano y de un tratamiento apropiado.

Descriptores: Embarazo, hipertensión arterial, síndrome de HELLP

Recibido: 4 de mayo de 2004

Aceptado: 05 de octubre de 2004

Correspondencia: Dr. Pablo Parra Ramírez Apto. 169-San Ramón. e-mail drpabparra@hotmail.com Dr.Mario Beckles Maxwell Apto. 10705-1000 San José. Abreviaturas: ALT, alanina aminotransferasa; AST, aspartato deshidrogenasa; CID, coagulación intravascular diseminada; CSD, cuadrante superior derecho; $\mathrm{DHL}$, deshidrogenasa láctica; GGT, gama glutamil transferasa; HCG, hormona gonadotropina coriónica humana; HIE, hipertensión inducida por el embarazo; HGAE, Hígado graso agudo del embarazo; HHSC, hematoma hepático subcapsular; $\mathrm{SH}$, Síndrome de HELLP;TP, tiempo de trombina; TPT, Tiempo de tromboplastina parcial.

ISSN 0001-6002/2005/47/1/7-14 Acta Médica Costarricense, (C2005 Colegio de Médicos y Cirujanos

\section{Generalidades y epidemiología}

En 1954 Pitchard y colaboradores describieron en tres mujeres embarazadas el cuadro clínico de lo que hoy se denomina $\mathrm{SH}^{1,2}$. Sin embargo, fue hasta 1982 con la descripción del doctor Louis Weinstein de 59 gestantes con alteraciones similares a las descritas por Pitchard cuando se consideró el cuadro como una variedad grave de la preeclampsia y eclampsia ${ }^{1,3-5}$.

Este autor acuñó los acrónimos HELLP en referencia a las principales alteraciones que se presentan en el cuadro clínico de estas pacientes, Dicho nombre corresponde a las siglas en inglés de "haemolysis, elevated liver enzymes and low platelets", las cuales en español corresponden a hemólisis, aumento de enzimas hepáticas y disminución de las plaquetas respectivamente.

Las enfermedades hipertensivas del embarazo afectan entre el 7 al $10 \%$ de todos los embarazos y contribuye al aumento de la morbimortalidad materna y perinatal ${ }^{6-10}$. En el caso del SH, este afecta al $0.1-0.6 \%$ de todas las gestaciones, del 4 al $20 \%$ de las preeclampsias y del $30-50 \%$ de las eclampsias ${ }^{1,3,4,11}$.

Se conoce que típicamente el SH va precedido de un cuadro de hipertensión y proteinuria, recientemente se han descrito $\mathrm{SH}$ en el seno de gestaciones normales, en alrededor del 15 al $20 \%$ no son precedidas por algún trastorno hipertensivo gestacional ${ }^{3}$. 
Este síndrome se ha descrito desde la mitad del segundo trimestre del embarazo hasta varios días posparto. Se dice que el $10 \%$ se producen antes de las 27 semanas, el $20 \%$ después de las 37 semanas y el $70 \%$ entre las 27 y las 37 semanas de gestación ${ }^{12,14}$.

\section{Diagnóstico y clasificación}

El diagnóstico de $\mathrm{SH}$ está basado en la evidencia de anemia hemolítica microangiopática, disfunción hepática y trombocitopenia en una mujer gestante o en su periodo posparto con o sin algún trastorno hipertensivo desarrollado ${ }^{14,15}$. Por ello, el diagnóstico de este síndrome es por exámenes de laboratorio y no directamente por las manifestaciones clínicas presentes, aunque éstas nos alertan de la severidad y de la evolución de la enfermedad.

El diagnóstico de hemólisis se ha basado en la presencia de anemia, hematíes fragmentados (esquistocitos), otros han incluido la presencia de cifras bajas de haptoglobina (normal 80-120 mg/dl), elevación de la deshidrogenasa láctica (DHL) mayor de $600 \mathrm{U} / 1$, bilirrubinas y hemoglobina libre1. Según Martín el aumento de la DHL y la disminución de las haptoglobinas séricas son los dos marcadores tempranos para el diagnóstico del SH, estos ocurren antes del incremento de los niveles de bilirrubina y antes de la disminución de la hemoglobina ${ }^{16}$. El inicio temprano en la disminución de las haptoglobinas sugiere que ocurre hemólisis y posteriormente inicia el proceso de la enfermedad.

En relación a la disfunción hepática, esta se evalúa principalmente con la elevación de la aspartato aminotransferasa (AST), alanina aminotransferasa (ALT) y DHL.

Por último la trombocitopenia, considerada como el tercer criterio diagnóstico esencial, es la más temprana anormalidad en la coagulación. Se consideran niveles diagnósticos de este síndrome menor de $150000 / \mathrm{mm}^{3}$.

El recuento plaquetario y los niveles de DHL son los mejores marcadores de la gravedad del proceso en la enfermedad ${ }^{16}$.

Sibai en 1993 elaboró los criterios diagnósticos para el SH y así mismo hizo la clasificación del tipo de Síndrome de HELLP (clasificación de Sibai o de Tennessee), la cual los cataloga como completo o incompleto. Los criterios diagnósticos para este síndrome son niveles de DHL mayor o igual a $600 \mathrm{U} / 1$, AST mayor o igual a $70 \mathrm{U} / 1$ y el recuento plaquetario menor o igual a $100000 / \mathrm{mm}^{3}$. El tipo de $\mathrm{SH}$ completo es aquel que tiene los tres parámetros y el incompleto es aquel que tiene al menos un parámetro de los antes señalados ${ }^{15,16}$.

Martín y colaboradores, en la Universidad de Mississippi, clasificaron el SH en tres clases en función del número de plaquetas, sabiendo que ante menor cantidad de plaquetas, la severidad del cuadro clínico y las complica- ciones son mayores. A esta se le llama clasificación de Martín o de Mississippi ${ }^{16,17}$ : Clase 1: Plaquetas menor de $50000 / \mathrm{mm}^{3}$; Clase 2: Plaquetas entre $50 \quad 000 \mathrm{y}$

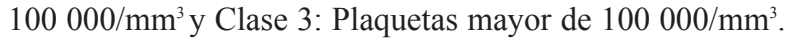

\section{Cuadro Clínico}

No existe ningún signo o síntoma que nos ayude a diferenciar el SH de la preeclampsia severa. El 70\% se presenta anteparto y el 30\% inicia en el puerperio, fundamentalmente en las primeras 48 horas. El 79\% de las pacientes que desarrollan $\mathrm{SH}$ en el posparto han sido diagnosticadas de preeclampsia en el anteparto ${ }^{4}$.

Generalmente las pacientes en riesgo de desarrollar $\mathrm{SH}$ son aquellas mayores de 25 años $^{14}$, blancas y multíparas, a diferencia de las pacientes que desarrollan preeclampsia o eclampsia que generalmente son de menor edad y primigestas.

Las manifestaciones clínicas son muy variadas y con frecuencia inespecíficas. Así la mayoría de las pacientes presentan dolor epigástrico o en hipocondrio derecho (65$90 \%$ ), malestar general (90\%), náuseas o vómitos (35-50\%) y cefalea $(30 \%)$ de pocos días de evolución. La aparición de síntomas de malestar general o "seudogripales" han sido considerados por algunos autores como la principal manifestación del cuadro ${ }^{18-21}$, por lo que se recomienda realizar un hemograma y determinación de enzimas hepáticas a toda gestante en el tercer trimestre de embarazo que inicie con estos síntomas inespecíficos, aun con valores normales de presión arterial ${ }^{21}$. Otras manifestaciones observadas con menos frecuencia son: convulsiones, ictericia, hemorragias (digestivas, urinarias, gingivales) y dolores musculares erráticos ${ }^{22,23}$.

En la exploración física es importante destacar que el $20 \%$ evolucionan sin hipertensión; el 30\% presentan hipertensión moderada (mayor o igual a 140/90 $\mathrm{mmHg}$ ) y el 50\% hipertensión grave (mayor o igual a 160/110 $\mathrm{mmHg}$ ). Por lo tanto los niveles de presión arterial no son diagnósticos del $\mathrm{SH}$, al igual que la proteinuria y los edemas, los cuales sí son importantes para el diagnóstico de preeclampsia, pero no para este síndrome ${ }^{3}$.

\section{Fisiopatología}

Al igual que en otras microangiopatías, el mayor componente de la perturbación en el síndrome de HELLP lo involucra el daño endotelial que se desarrolla en esta patología $^{3}$.

Las diferentes teorías propuestas comparten que el inicio del proceso de la enfermedad es el daño en la capa íntima endotelial, causado por mecanismos aún inciertos pero que se ha asociado a alteraciones en la placentación, complejos inmunes y a la misma hipertensión, en todo caso lo que se produce es una injuria endotelial donde se estimula el depósito de fibrina en el lumen vascular con subsecuente ruptura de los elementos de la sangre por el contacto con el área daña- 
da. Esto producirá un desequilibrio entre las sustancias vasodilatadoras (prostaciclina) y vasoconstrictoras (Tromboxano A2, serotonina, endotelina-1) a favor de éstas últimas que tendrían un papel fundamental en la patogenia del proceso al producir una activación del sistema de la coagulación, con consumo de plaquetas y provocar además un espasmo vascular en la microcirculación con trombosis arterial, hipertensión y reducción del flujo uteroplacentario ${ }^{1}$.La ruptura del equilibrio entre sustancias vasodilatadoras y vasoconstrictoras, junto con el descenso del óxido nítrico (vasodilatador) liberado por el endotelio hace que la aglutinación y la agregación de plaquetas a la lesión endotelial sea cada vez mayor, liberando éstas a su vez más tromboxano A2 y serotonina, creando un círculo vicioso que hasta ahora sólo se sabe que se rompe con la evacuación del útero ${ }^{2}$.

La consecuencia es un consumo de plaquetas acelerado, con aparición de microtrombos y depósitos de fibrina en los distintos órganos con afectación endotelial (riñon, hígado y principalmente útero). El aumento de los megacariocitos hallados en las biopsias de médula confirma ésta hipótesis ${ }^{4}$.

En la preeclampsia la vida media de las plaquetas se reduce de 3-5 días (normal 10 días). En el SH disminuye aún más la vida media y la integridad estructural de estas plaquetas, dando así agregación y destrucción de las mismas ${ }^{16}$.

La alteración en la membrana de las plaquetas en el SH libera ácido araquidónico y otras aminas vasoactivas causando una fuerte vasoconstricción y vasoespasmos, acelerando aún más la agregación y destrucción plaquetaria. La disminución de las plaquetas significa que el efecto compensador de la médula ósea no es suficiente para solventar las necesidades ${ }^{16}$. Se ha observado que la Beta-trombomodulina, la cual es una proteína específica de las plaquetas, aumenta cuando estas son agregadas. Su elevación es debida a la agregación plaquetaria con consumo de la microvasculatura y a la disminución de la aclaración renal ${ }^{16}$.

La activación de la coagulación y de la fibrinólisis puede llevar a la aparición de una coagulación intravascular diseminada (CID), lo que agrava la trombocitopenia. Como mencionamos anteriormente la lesión endotelial causa ruptura de los elementos formes de la sangre, principalmente de los glóbulos rojos los cuales se fragmentan por el paso a rápida velocidad por el endotelio dañado, manifestándose en esquistocitos y otras formas en el frotis de sangre periférica y en anemia hemolítica microangiopática, la cual parece ser el reflejo del compromiso de los glóbulos rojos y de la disfunción endotelial ${ }^{4}$.

En el SH se entiende el proceso fisiopatológico que ocurre, pero aún quedan inciertos los mecanismos por los cuales se desencadenan.
En el SH puede haber un serio compromiso hepático con eventual hemorragia subcapsular y raramente una catastrófica ruptura hepática.

El compromiso se debe al depósito de fibrina a nivel de las sinusoides hepáticas pudiendo llevar a una necrosis periportal que coalesce y se diseca dentro de la cápsula de Glisson ${ }^{16}$.

En la biopsia hepática encontramos la típica lesión histológica que se asocia con el $\mathrm{SH}$ : necrosis del parénquima periportal con depósitos de fibrina en el espacio sinusoidal, que puede ser el responsable de la elevación de las enzimas hepáticas. Se pueden encontrar microtrombos en el espacio sinusoidal de las zonas necrosadas y del parénquima sano. Estos depósitos de fibrina obstaculizan el flujo sanguíneo hepático, distendiendo el hígado. La tensión provocada en la cápsula de Glisson puede originar el dolor en epigastrio y en hipocondrio derecho ${ }^{18,24,25}$.

Si la afectación del hígado es masiva podemos encontrar una hemorragia hepática y/o hematoma subcapsular, el cual puede posteriormente sufrir ruptura y dar un cuadro catastrófico ${ }^{26-28}$.

La ecografía hepática puede poner de manifiesto áreas hipoecogénicas perihepáticas correspondientes a un hematoma subcapsular ${ }^{4}$.

\section{Diagnóstico Diferencial}

El diagnóstico diferencial de las pacientes con $\mathrm{SH}$ debe incluir gran variedad de procesos. No obstante, los cuadros más importantes con los que hay que diferenciarlos son aquellos que cursan con trombocitopenia (trombocitopenia gestacional, púrpura trombocitopénica autoinmune), o bien asociada con anemia hemolítica microangiopática (preeclampsia, síndrome hemolítico urémico, púrpura trombótica trombocitopénica, hígado graso agudo del embarazo).

\section{Complicaciones del Síndrome de HELLP}

\section{Complicaciones Maternas}

Este síndrome cursa con alta morbimortalidad materna, aunque varía entre $0-24 \%$, dependiendo fundamentalmente de la precocidad con que se realice el diagnóstico ${ }^{29}$. Pacientes con SH tienen mayor morbimortalidad materna y perinatal en comparación con pacientes con preeclampsia severa sin SH. La morbilidad se asocia a CID, abruptio placentae, insuficiencia renal aguda, edema pulmonar, hematoma hepático subcapsular y desprendimiento de retina ${ }^{26-30}$. 


\section{Complicaciones Fetales}

La mortalidad perinatal es muy elevada y como se mencionó anteriormente varía entre 30 a 40\%. Las causas principales de esta elevada mortalidad son la prematuridad, la abruptio placentae y el sufrimiento fetal intraútero ${ }^{31}$.

Otras complicaciones que se han reportado es que los infantes pueden tener leucopenia entre el 9.7 y $38 \%$ y trombocitopenia entre 26 y 34\%. Sin embargo, no existe correlación entre los hallazgos hematológicos de la madre y el recién nacido ${ }^{32}$.

\section{Manejo del síndrome de HELLP}

El embarazo complicado con $\mathrm{SH}$ requiere de un reconocimiento temprano de la enfermedad y de la institución de una terapia adecuada. Todo el siguiente texto está basado en la experiencia clínica de el Centro Médico de la Universidad de Mississipi ${ }^{16}$, los cuáles crearon un esquema de abordaje óptimo de pacientes con SH que consta de 12 pasos, solamente se tomaron otras bibliografías para ampliar el manejo, las cuales se indican en su momento respectivo:

\section{1) Diagnóstico temprano:}

Para hacer el diagnóstico de SH lo primero es tener presente ésta enfermedad en toda paciente embarazada y en especial en toda paciente con gestosis. Si se sospecha el diagnóstico de SH está indicado realizar las pruebas de laboratorio básicas para tal diagnóstico (plaquetas, transaminasas, DHL) ${ }^{32}$.

En tempranas fases, el SH puede exhibir únicamente un modesto incremento en la DHL, AST y ALT, y una trombocitopenia clase $3^{34,35}$.

Aunque el SH es una variante de la preeclampsia severa, el diagnóstico puede quedar incierto en la mujer con hipertensión arterial y proteinuria hasta que las plaquetas sean menor de $100000 / \mathrm{mm}^{3}$ y la DHL mayor de $600 \mathrm{U} / 1^{35}$.

Cabe resaltar para la sospecha del $\mathrm{SH}$, que la preeclampsia es un desorden de mujeres jóvenes y nulíparas, pero el SH es de mujeres añosas y multíparas.

Existen factores clínicos de riesgo que son fáciles de identificar en la paciente, como náuseas, vómitos y dolor en epigastrio, los cuales nos pueden alertar sobre la condición materna y su posible evolución si no se trata a tiempo. Las pacientes con evidencia de preeclampsia, dolor en cuadrante superior derecho y náuseas deben ser seriamente evaluadas como potenciales SH. La ausencia de hipertensión arterial diastólica puede ser malinterpretado como un signo tranquilizador del diagnóstico de posible preeclampsia con $\mathrm{SH}$, sin embargo recordemos que un cierto porcentaje de pacientes cursan con cifras de presión arterial entre los parámetros normales.
En la paciente con hipertensión arterial, dolor epigástrico y hemólisis, la aparición de anormalidades en el ojo podrían advertir al médico para la sospecha de un $\mathrm{SH}$. Como por ejemplo hallazgos oculares de hemorragia vítrea, desprendimiento de retina, entre otros.

Un reciente grupo identificado con riesgo para el desarrollo de $\mathrm{SH}$ son aquellas con embarazos en el segundo trimestre con aumento de la alfa fetoproteína en suero y elevación de la hormona gonadotropina coriónica humana (HCG). Se ha observado que mujeres con elevación de ambos marcadores séricos tienen 47 veces más riesgo de desarrollar SH.

\section{2) Valoración de la condición materna:}

La valoración inicial de laboratorio de pacientes con preeclampsia o sospecha de SH debe incluir un hemograma completo, enzimas hepáticas, pruebas de función renal, ácido úrico y proteinuria. La presencia de trombocitopenia, menor de $150000 / \mathrm{mm}^{3}$, requiere de una investigación más cuidadosa. En ausencia de otras manifestaciones de $\mathrm{SH}$, como por ejemplo anemia hemolítica microangiopática y disfunción hepática, se debe de hacer diagnóstico diferencial de trombocitopenia en el embarazo. Una elevación de DHL en el SH es una manifestación tanto de la anemia hemolítica microangiopática como de la disfunción hepática que se desarrolla en este proceso patológico. La disfunción hepática es también manifestada por incremento variable de las transaminasas, AST y ALT. Una DHL que excede los $600 \mathrm{U} / 1$ es necesario para el diagnóstico del síndrome y se predice una seria morbilidad con valores ma-yores de 1 $400 \mathrm{U} / 1^{35}$.

El primer trastorno de la coagulación que se desarrolla en el SH es la trombocitopenia, seguida más tarde de aumento del fibrinógeno y sus productos de degradación y por último una CID.

Otro factor de riesgo para la morbilidad materna es un ácido úrico mayor de $7.8 \mathrm{mg} / \mathrm{dl}$, por lo que se debe indicar en toda paciente con $\mathrm{SH}$, pues nos ayudará a predecir el pronóstico de la paciente sino actuamos adecuadamente.

El laboratorio básico en toda paciente con sospecha de $\mathrm{SH}$ es el siguiente: hemograma completo con niveles de plaquetas, análisis de orina, creatinina sérica, deshidrogenasa Láctica, ácido úrico, bilirrubina indirecta y total, AST y ALT. El TP, TPT, fibrinógeno y productos de degradación del fibrinógeno sólo se indican en pacientes con un recuento plaquetario menor de $100000 / \mathrm{mm}^{3}$. Los electrolitos y glucosa no son usualmente necesarios ${ }^{35}$.

Se debe de valorar seriamente los valores de plaquetas, DHL y enzimas hepáticas cada 12-24 horas o más frecuentemente si la clínica lo amerita. 


\section{3) Valoración de la condición fetal:}

El SH es una variante atípica de la preeclampsia severa $\mathrm{y}$, como tal, el único tratamiento es la interrupción del embarazo y la remoción de los factores citotóxicos que la produce. El momento del parto requiere de varios factores, incluyendo la severidad de la condición materna, condición fetal y placenta, y la edad gestacional.

La condición fetal debe ser evaluada mediante una prueba no estresante, una prueba de contracción estresante y/o un perfil biofísico fetal. Además, en la actualidad, la velocimetría Doppler de la circulación útero y fetoplacentaria puede utilizarse para evaluar complicaciones asociadas al retardo de crecimiento intrauterino y otras formas de sufrimiento fetal debido a hipoxemia o asfixia como el producido por los trastornos hipertensivos del embarazo ${ }^{36}$. También pueden diagnosticarse anomalías cardíacas fetales y otras malformaciones, y alteraciones placentarias ó del cordón umbilical. La ultrasonografía Doppler es una de las herramientas clínicas más importantes para la vigilancia de fetos que sufren trastornos hipertensivos del embarazo. Un aumento en los índices de los vasos uterinos o umbilicales puede mostrar una alteración en la circulación placentaria. Varios estudios han sugerido que el estudio Doppler de la arteria uterina puede ser útil como una herramienta de screening para detectar precozmente aquellos embarazos que sufrirán trastornos hipertensivos ${ }^{37}$. La ultrasonografía Doppler se presenta como un estudio inocuo tanto para la madre como para el feto, no invasivo, rápido y repetible, que brinda importante información acerca del estado hemodinámico fetal y permite un seguimiento perinatal exhaustivo para disminuir la morbimortalidad asociada a hipoxia crónica, evitando la descompensación secundaria a stress. Sin embargo, su uso en embarazos de bajo riesgo no ha demostrado ser útil como herramienta de screening, excepto en la detección precoz de trastornos hipertensivos ${ }^{37}$. Por su parte, el estado materno, como lo mencionamos anteriormente, se determina por historia clínica, examen físico y pruebas de laboratorio.

Con pocas excepciones, embarazos mayores de 34 semanas y clase 1 del SH son interrumpidos en 24 horas, vía vaginal o cesárea. Embarazos entre 24 y 34 semanas con riesgo de pretérmino deben administrárseles terapia con corticoesteroides para acelerar la madurez pulmonar fetal. También se ha observado un beneficio materno con la administración de corticoesteroides, pues incrementa o estabiliza las plaquetas, desciende o estabiliza la DHL, AST y ALT $^{34}$.

Los beneficios de esta terapia en neonatos son reducción de los días de estancia en la unidad de cuidados neonatales, menos incidencia de hemorragia intraventricular, de enterocolitis necrotizante, menos fibroplasia retrolental y menos mortalidad neonatal.
Todo SH con tratamiento anteparto con altas dosis de corticoesteroides requieren también de su administración en el posparto para prevenir el rebote de plaquetas. Si estas pacientes no reciben corticoesteroides posparto el fenómeno de rebote se caracteriza por aumento de la DHL, AST y ALT, severa trombocitopenia y oliguria.

\section{4) Control de la presión arterial:}

Entre el $80-85 \%$ de las pacientes con SH desarrollan cifras elevadas de presión arterial. La presión arterial se debe bajar para prevenir las complicaciones maternas y posibles riesgos de abrupción de placenta y alteración en su perfusión.

Se debe administrar tratamiento antihipertensivo cuando la presión arterial sistólica se encuentre por encima de los $150 \mathrm{mmHg}$ y si la diastólica es mayor o igual a 100 $\mathrm{mmHg}$. Entre los fármacos que se pueden utilizar durante el embarazo está la hidralazina, el cual es un vasodilatador arterial y es usado en el manejo agudo de la hipertensión en el SH. Se administra en una dosis de 5-10 mg en bolo intravenoso con una frecuencia de 20-40 minutos. Si la hidralazina es inefectiva o contraindicada se puede utilizar otros fármacos como labetalol y nitroprusiato de sodio. El labetalol se utiliza en dosis de $20 \mathrm{mg}$ en bolo intravenoso, aumentado la dosis progresivamente hasta una presión arterial satisfactoria (máximo $300 \mathrm{mg}$ ). Por su parte, el nitroprusiato de sodio, un potente vasodilatador arterial y venoso, se inicia con una dosis de $0.25 \mathrm{ug} / \mathrm{Kg} /$ minuto y puede ser incrementado hasta $10 \mathrm{ug} / \mathrm{Kg} /$ minuto ${ }^{34}$. Un agente antihipertensivo ideal para ser usado en el posparto es la nifedipina, un calcioantagonista con propiedades de vasodilatador arterial periférico, el cual con su administración vía oral y no sublingual, ha mostrado tener múltiples efectos beneficiosos como disminuir la presión arterial, restaurar la diuresis e inclusive normalizar las plaquetas en el posparto.

\section{5) Prevención de las convulsiones con sulfato de magnesio:}

Las convulsiones eclámpticas frecuentemente preceden o siguen al desarrollo del SH, por tal motivo es recomendable que toda paciente con SH (especialmente aquella cerca de la labor de parto o con dolor epigástrico) reciba infusión intravenosa de sulfato de magnesio de 4-6 g en bolo, seguida de una infusión constante de 1.5-4 g /hora. Se debe de monitorizar a las pacientes con el reflejo patelar, diuresis y niveles séricos de magnesio. Se puede continuar su administración hasta 48 horas posparto, dependiendo de la condición materna.

Además de prevenir y de tratar las convulsiones eclámpticas, el sulfato de magnesio tiene propiedades de ser un modesto relajante vascular tanto a nivel central como periférico. 
En raras ocasiones está contraindicado el uso de sulfato de magnesio, ejemplo de ello son las pacientes con miastenia gravis, donde la droga de escogencia es la fenitoína. Se administra en dosis de $15 \mathrm{mg} / \mathrm{kg}$, dado a razón de 40 $\mathrm{mg} /$ minuto con continuo monitoreo cardiaco y de la presión arterial cada 5 minutos. La dosis terapéutica de la fenitoína es de $10-20 \mathrm{ug} / \mathrm{ml}$.

\section{6) Manejo de los fluidos y electrolitos:}

La combinación de vasoespasmo y daño endotelial en pacientes con $\mathrm{SH}$ causa excesos o deficiencias intravasculares. Un régimen recomendado para el manejo de fluidos y electrolitos en pacientes con $\mathrm{SH}$ es alternar 5\% de dextrosa y $500 \mathrm{ml}$ de suero salino normal y $5 \%$ de solución de lactato de ringer a razón de $100 \mathrm{ml} /$ hora para mantener una diuresis de al menos $20 \mathrm{ml} /$ hora (preferiblemente 30-40 $\mathrm{ml} /$ hora). El control debe ser estricto pues el exceso de fluidos puede exacerbar una vasoconstricción con daño renal y daño pulmonar con edema pulmonar cardiogénico.

En pacientes oligúricas, uno o dos bolos de solución salina o 250-500 $\mathrm{ml}$ pueden ser administrados para restaurar la diuresis. Si la oliguria persiste, el estado del volumen intravascular debe ser monitorizado con la presión en cuña de los capilares pulmonares usando un catéter de Swan-Ganz.

\section{7) Hemoterapia:}

La hemorragia espontánea en pacientes con SH puede ocurrir desde poner vías intravenosas hasta incisiones quirúrgicas cuando las plaquetas están menores de $50000 / \mathrm{mm}^{3}$. La transfusión de plaquetas está recomendada en mujeres con preeclampsia severa que van para cesárea y que su recuento plaquetario sea inferior a $50000 / \mathrm{mm}^{3}$. Las plaquetas pueden ser transfundidas justo antes de la incisión abdominal si las plaquetas están menores de 40 000/ $\mathrm{mm}^{3} \mathrm{o}$ se pueden esperar hasta más tarde en la cirugía y administrar las plaquetas únicamente si hay excesiva hemorragia.

En pacientes con $\mathrm{SH}$ que van a parto vaginal se deben transfundir plaquetas si el recuento plaquetario es menor de $20000 / \mathrm{mm}^{3}$. Después del parto es recomendable la transfusión de plaquetas en las primeras 24 horas posparto para mantener las plaquetas por encima de $50000 / \mathrm{mm}^{3}$ en cesárea y mayor de $20000 / \mathrm{mm}^{3}$ en parto vaginal para prevenir la formación de un hematoma. En la práctica diaria una transfusión de plaquetas generalmente implica la administración de 4 a 5 unidades, con lo que se espera un incremento en el recuento plaquetario de aproximadamente 20000 a 25000 plaquetas $/ \mathrm{mm}^{3}{ }^{38}$.

El uso de dexametasona para el tratamiento de pacientes con síndrome de HELLP con plaquetas menores de $100000 / \mathrm{mm}^{3}$ ha eliminado la necesidad del uso de la transfusión de plaquetas.

\section{8) Manejo de la labor y del parto:}

Se debe de realizar una valoración cuidadosa del estado materno y fetal. El inmediato uso de dexametasona, a dosis de $10 \mathrm{mg}$ cada 12 horas IV, tan pronto como se hace el diagnóstico de $\mathrm{SH}$ ha mostrado hacer en gestaciones de pretérmino dos funciones: 1 . Acelerar la madurez pulmonar fetal si el parto va a ocurrir en menos de 24-48 horas. 2. Mejora el proceso de la enfermedad materna al permitir una mejor madurez cervical y la inducción de la labor de parto.

Algunos estudios recientes revelan un mayor número de partos vaginales en pacientes con SH en las cuales se usó altas dosis de dexametasona y también se determinó una mejoría en el proceso de la enfermedad.

Si se requiere una cesárea, una incisión de Pfannenstiel es la mejor opción, debido a que está relacionada con menos casos de ruptura e infección de la herida quirúrgica ${ }^{16}$. Sin embargo según la experiencia de ginecólogos de nuestro medio, la incisión media se prefiere ya que se producen menos hematomas que con la incisión de Pfannenstiel.

Se deben dar un curso corto de antibióticos (24-48 horas) si los productos sanguíneos fueron administrados, porque se ha encontrado mayor incidencia de infecciones en pacientes con SH que recibieron transfusión sanguínea, en comparación con las que no recibieron transfusión.

En cuanto a la anestesia que se le puede administrar a estas pacientes, la epidural puede ser administrada seguramente en pacientes sin hemorragia ni secuelas neurológicas, si las plaquetas están por encima de $100 \quad 000 / \mathrm{mm}^{3}$. La anestesia general ha tenido complicaciones en pacientes con SH y daño en su hígado, debido a que no se metaboliza adecuadamente el agente anestésico empleado.

Es importante que en el periodo periparto se vigile adecuadamente a las pacientes por hemorragia hepática y posible ruptura del mismo. Se debe tener presente la tríada de la ruptura hepática: Paciente con preeclampsia, eclampsia o $\mathrm{SH}$, dolor en cuadrante superior derecho e hipotensión súbita. Cuando existe hemorragia hepática el dolor en epigastrio y en cuadrante superior derecho inician progresivamente y empeoran con irradiación a la espalda por colapso vascular, shock y signos de hemoperitoneo.

Durante la cesárea no es recomendable la valoración del hígado, debido a que existe riesgo de una ruptura traumática de un hematoma subcapsular. El mejor tratamiento en caso de sangrado hepático es la embolización arterial hecha por un cirujano experimentado.

\section{9) Cuidado Perinatal Óptimo:}

El principal riesgo fetal en un embarazo complicado con $\mathrm{SH}$ es la prematuridad. Por tal motivo, la administración de un corticoesteroide a la madre puede ser usado para acelerar la madurez fetal pulmonar y para disminuir el 
riesgo de enterocolitis necrotizante y hemorragia intraventricular en productos de embarazos de 24 a 34 semanas de gestación.

Es recomendable la valoración de rutina de las plaquetas neonatales en recién nacidos de madres con $\mathrm{SH}$, porque se ha encontrado asociación entre la plaquetopenia materna con aumento en el riesgo de hemorragia intraventricular en el feto. La inestabilidad cardiorrespiratoria neonatal se ve más en embarazos con SH con partos antes de las 32 semanas de gestación.

\section{0) Tratamiento Intensivo en el posparto:}

El SH se puede manifestar primariamente en el periodo posparto o seguir manifestándose aún con la interrupción del embarazo. Es recomendado que todas las pacientes con preeclampsia-SH sean tratadas en un salón de recuperación obstétrica que funciona como una unidad intermedia de cuidados intensivos por tanto tiempo como: Las plaquetas tengan un ascenso constante y la DHL un descenso constante; la paciente tenga una diuresis de más de $100 \mathrm{ml} /$ hora por 2 horas consecutivas sin el uso de fluidos o diuréticos, hipertensión arterial debe estar controlada (PA sistólica en $150 \mathrm{mmHg}$ y la diastólica en menos de $100 \mathrm{mmHg}$ ); la paciente tenga una mejoría clínica obvia y que no tenga riesgo significativo de complicaciones.

Se deben valorar las plaquetas y los niveles de DHL continuamente cada 12 horas hasta que la paciente se le externe. El sulfato de magnesio se debe continuar hasta que el cuadro de preeclampsia, eclampsia o $\mathrm{SH}$ esté resulto o al menos por 24 horas posparto o poscirugía. La evaluación del TP, TPT y fibrinógeno no es necesario al menos que las plaquetas maternas estén menor de $50000 / \mathrm{mm}^{3}$ o se evidencie una coagulopatía de consumo.

Se deben de usar los corticoesteroides en el posparto porque estos ayudan a resolver más rápidamente el cuadro, incrementando la diuresis, las plaquetas, disminuyendo la presión arterial media, la DHL y AST, y con ello disminuye el riesgo de complicaciones maternas. Se inicia la administración de corticoesteroides hasta las 12 horas posparto, en dosis de $10 \mathrm{mg}$ de dexametasona cada 12 horas dos dosis, luego se baja la dosis a $5 \mathrm{mg}$ cada 12 horas dos dosis. También existe otro esquema de tratamiento posparto que dice que se debe administrar dexametasona $10 \mathrm{mg}$ cada 12

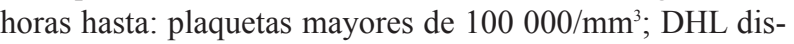
minuida; diuresis mayor de $100 \mathrm{ml} /$ hora; clínica estable.

Si la paciente cumple con estos 4 criterios, se administrarán $5 \mathrm{mg}$ de dexametasona cada 12 horas dos dosis y luego se egresa. En el cuadro 1 podemos observar un resumen del manejo con dexametasona.

Se ha observado una más rápida resolución del cuadro de preeclampsia/eclampsia con la remoción quirúrgica de algunos remanentes de tejido decidual por dilatación y curetaje. Recientemente se hace lo mismo pero guiado por ultra-
Cuadro 1. Uso de dexametasona en el síndrome de HELLP

\section{Anteparto: $10 \mathrm{mg} \mathrm{IV/} \mathrm{c/12} \mathrm{horas}$}

Cuando las plaquetas sean menor de 100 000/.mm

Si las plaquetas están entre $100000-150$ 000/mm

Eclampsia, dolor epigástrico, hipertensión severa o enfermedad fulminante.

\section{Posparto: $10 \mathrm{mg}$ IV c/12hras \#2, luego $5 \mathrm{mg}$ IV} c/12hrs \#2 dosis

Cuando se usó esteroides en el anteparto

Paciente recobrando niveles adecuados de plaquetas y de DHL

Si la condición clínica lo amerita

sonido. También se ha observado que la administración oral de $10 \mathrm{mg}$ de nifedipina cada 4 horas por las primeras 48 horas posparto en pacientes con preeclampsia severa ha tenido resultados efectivos iguales a los del curetaje posparto en disminuir la presión arterial media y en incrementar la diuresis.

Si la paciente no responde al tratamiento con dexametasona en las primeras 72 horas después de administrado, se recomienda la plasmaféresis.

\section{1) Alerta en el desarrollo de fallo multiorgánico:}

Las pacientes con severo dolor epigástrico pueden estar en riesgo aumentado de hemorragia hepática o ruptura. Si la ruptura ocurre el único tratamiento es quirúrgico. Pacientes con SH y un volumen sanguíneo contraído tienen mayor riesgo de desarrollar una insuficiencia renal aguda, insuficiencia pulmonar aguda y síndrome de distress respiratorio. En estos casos, el rápido reemplazo del volumen intravascular con sangre y productos sanguíneos es necesario para bloquear el compromiso alveolar y glomerular. En pacientes con fallo multiorgánico, la transfusión de plasma facilita la resolución de esta complicación.

\section{2) Consejo sobre futuros embarazos:}

El riesgo de recurrencia de una preeclampsia y eclampsia es del 42-43\% y del SH es de 19-27\%. Si el embarazo previo finalizó antes de las 32 semanas de gestación, la recurrencia de un parto de pretérmino en el próximo embarazo es del $61 \%$.

\section{Abstract}

The HELLP syndrome of haemolysis, elevated liver enzymes and low platelets is a major complication of pregnancy. Ten percent of pregnancies complicated by severe preeclampsia and eclampsia are affected by HELLP syndrome. 
HELLP syndrome has been described as firts appearing from the mid second trimester of pregnancy until severe days postpartum. Approximately one third of patients will have their first manifestation of HELLP syndrome diagnosed postpartum. Of the two thirds of women who are first diagnosed with HELLP syndrome antepartum, 10\% will be identified before 27 weeks, $20 \%$ in pregnacies beyond 37 weeks, and the majority, 70\%, occurring between 27 and 37 weeks gestation. The maternal mortality has been reported to be as high as $24 \%$ and perinatal mortality can range as high as $30-40 \%$.

The early diagnosis and treatment of HELLP syndrome remains problematic for the obstetric health care provider. The non-especific signs and symptoms of this disorder early in the disease process make the accurate diagnosis difficult and delays early treatment, which has the best prognosis for both maternal and perinatal outcome. The management of the pregnancy complicated by HELLP syndrome requires early recognition and the institution of the appropriate therapy.

\section{Referencias}

De la Rubia, J. Síndrome HELLP. Med Clin 2001;117: 64-68.

2. Pritchard JA, Weisman R, Ratnoff OD, Vosburgh GJ. Intravascular hemolysis, trombocytopenia, and other hematologic abnormalities associated with severe toxemia of pregnancy. N Engl J Med 1954; 250:89-98.

3. Sibai, B. HELLP Syndrome. Clin Obstet Gynecol.1999;42:381-389.

4. Sibai, B. Velasco, J. El Síndrome de HELLP: Una complicación de la Preeclampsia. Clin Invest Gynecol Obstet. 1994;21:133-137.

5. Weinstein L. Syndrome of hemolysis, elevated liver enzymes and low platelet count: a severe consequense of hypertension in pregnancy. Am J Obstet Gynecol 1982; 142:159-167.

6. Gilabert J, Estellés A, Ridocci F, España F, Aznar J, Galbis M. Clinical and haemostatic parameters in the HELLP syndrome: relevance of plasminogen activator inhibitors. Gynecol Obstet Invest 1990;30:81-86

7. Jiménez, Edwin. Guías para la detección, diagnóstico y tratamiento de la hipertensión arterial en el primer nivel de atención. Costa Rica: CCSS, 2002.

8. Martin JN Jr, Blake PG,Lowry SL, Perry KG Jr, McCaul JF, Hess LW, Martin RW. The natural history of HELLP syndrome: Patterns of disease progression and regression. Am J Obstet Gynecol 1991;164:1500-1513.

9. Goodlin RC. HELLP does not always mean inmediate HELP! Am J Obstet Gynecol 1990;163:1089.

10. Sullivan CA. The recurrence risk of the HELP syndrome in subsequent gestations. Am J Obstet Gynecol 1994;171:940-943

11. Fish R. The HELLP Syndrome:case report and review of the literature. J Emer Med 1993;11:169-174

12. Sibai BM. The HELLP syndrome: much ado about nothing? Am J Obstet Gynecol 1990; 162:311-316

13. Sibai BM, Ramadan MK, Usta I, Salama M, Mercer BM, Friedman SA. Maternal morbidity and mortality in 442 pregnancies with hemolysis, elevated liver enzymes and low platelet. Am J Obstet Gynecol 1993;169:1000-1006

14. Sibai BM, Taslimi MM, El-Nazer A. Maternal-perinatal outcome associated with the HELLP Syndrome in severe preeclampsiaeclampsia. Am J Obstet Gynecol 1986;155:501-509

15. Audibert F, Friedman S, Frangie A, Sibai BM. Clinical utility of strict diagnostic criteria for the HELLP syndrome. Am J Obstet Gynecol 1996; 175:460-464

16. Martín, J. Twelve steps to optimal management of HELLP syndrome. Clin Obstet Gynecol.1999;42:532-550.

17. Martín J. Pregnancy complicated by preeclampsia/eclampsia with the HELLP syndrome. How rapid is pospartum recovery? Obstet Gynecol. 1990;76:737-741

18. Barton JR, Riely CA, Adamec TA, Shanklin DR, Khoury AD, Sibai BM. Hepatic histopathologic condition does not correlate with laboratory abnormalities in HELLP syndrome. Am J Obstet Gynecol 1992;167:1538-1543.

19. Barton JR, Sibai BM. Care of the pregnancy complicated by HELLP syndrome. Obstet Gynecol Clin North Am 1991;18:165-179

20. Reubinoff BE. HELLP syndrome complicating preeclampsiaeclampsia. Int J Gynecol Obstet 1991;36:95-102

21. Weinstein L. Preeclampsia/eclampsia with hemolysis, elevated liver enzymes and low platelets. Obstet Gynecol 1985;66:657-660

22. McCrae KR. Thrombotic microangiopathy during pregnancy. Semin Hematol 1997;34:148-158.

23. Stone JH. HELLP syndrome. JAMA 1998;20:559-562.

24. Burrow, G. Medical Complication During Pregnancy. 5o edición. EEUU: Saunders, 1999;212

25. Knapen, M. Liver function following pregnancy complicated by the HELLP syndrome. Br J Obstet Gynecol 1998;105:1208-1210.

26. Nelson EW, Archibald L, Albo D. Spontaneous hepatic rupture in pregnancy. Am J Surg. 1977;134:817-820

27. Van Dyke RW. The liver in pregnancy. In: Zakim D, Boyer TD, editors. Hepatology: a textbook of liver disease. $2 \mathrm{~d}$ ed. Philadelphia: WB Saunders; 1990. p 1438.

28. Westergaard L. Spontaneous rupture of the liver in pregnancy. NY St J Med 1980;84:314.

29. Ravetti, O. Síndrome HELLP: Una complicación de la preeclampsia. Revista de Posgrado de la Cátedra VI año de Medicina.2001;103.2931.

30. Martín J. HELLP Syndrome: currents principles and recommended practices. Curr Obstet Med 1996;4:129-175

31. Saphier CJ, Repke JT et al. The HELLP syndrome: a review of diagnosis and management. Semin Perinatol 1998;22:118-133.

32. Tomsen TR. HELLP syndrome presenting as generalized malaise. Am J Obstet Gynecol. 1995;172:1876-1878

33. Martín JN Jr,Magna EF, Blake PG. Analysis of 454 pregnacies with severe preeclampsia/eclampsia HELLP syndrome using the 3-class system of classification. Am J Obstet Gynecol. 1993;68:386

34. Martín, J. The espectrum of severe preeclamspia: Comparative analysis by HELLP syndrome. Am J Obstet Gynecol.1999;180:1373-1384.

35. Geary M. The HELLP Syndrome. Br J Obstet Gynecol 1997; 104:887-89

36. Bower S, Bewley S, Campbell S. Improved prediction of pre-eclampsia by two-stage screening of uterine arteries using the early diastolic notch and color Doppler imaging. Obstet Gynecol 1993; 82: 78-83.

37. Alfirievic Z, Nielson JP. Doppler ultrasonography in high-risk pregnancies: systemic review with meta-analysis. Am J Obstet Gynecol 1995; 172: 1379-1387.

38. Strauss RG. Economy of platelet transfusions from a hospital perspective: pricing predicates practice. Transfusion. 2001;41:1617-24. 